DOI: $10.17516 / 1997-1397-2021-14-5-604-610$

УДК 512.54

\title{
On Pairs of Additive Subgroups Associated with Intermediate Subgroups of Groups of Lie Type over Nonperfect Fields
}

\author{
Yakov N. Nuzhin* \\ Siberian Federal University \\ Krasnoyarsk, Russian Federation
}

Received 06.03.2021, received in revised form 20.04.2021, accepted 24.6.2021

\begin{abstract}
The author has previously (Trudy IMM UrO RAN, 19(2013), no. 3) described the groups lying between twisted Chevalley groups $G(K)$ and $G(F)$ of type ${ }^{2} A_{l},{ }^{2} D_{l},{ }^{2} E_{6},{ }^{3} D_{4}$ in the case when the larger field $F$ is an algebraic extension of the smaller nonperfect field $K$ of exceptional characteristic for the group $G(F)$ (characteristics 2 and 3 for the type ${ }^{3} D_{4}$ and only 2 for other types). It turned out that apart from, perhaps, the type ${ }^{2} D_{l}$, such intermediate subgroups are standard, that is, they are exhausted by the groups $G(P) H$ for some intermediate subfield $P, K \subseteq P \subseteq F$, and of the diagonal subgroup $H$ normalizing the group $G(P)$. In this note, it is established that intermediate subgroups are also standard for the type ${ }^{2} D_{l}$.
\end{abstract}

Keywords: groups of Lie type, nonperfect field, intermediate subgroups, carpet of additive subgroups.

Citation: Ya.N. Nuzhin, On Pairs of Additive Subgroups Associated with Intermediate Subgroups of Groups of Lie Type over Nonperfect Fields, J. Sib. Fed. Univ. Math. Phys., 2021, 14(5), 604-610.

DOI: $10.17516 / 1997-1397-2021-14-5-604-610$.

\section{Introduction and preliminaries}

Groups of Lie type $G(F)$ over the field $F$ consist of Chevalley groups of type $\Phi=A_{l}, B_{l}$, $C_{l}, D_{l}, E_{6}, E_{7}, E_{8}, F_{4}, G_{2}$ and twisted Chevalley groups of type ${ }^{n} \Phi={ }^{2} A_{l},{ }^{2} D_{l},{ }^{3} D_{4},{ }^{2} E_{6},{ }^{2} B_{2}$, ${ }^{2} G_{2},{ }^{2} F_{4}$. The number of fundamental reflections generating the Weyl group associated with the group $G(F)$ is called its Lie rank. Groups of type $A_{1},{ }^{2} A_{2},{ }^{2} B_{2},{ }^{2} G_{2}$ constitute all groups of Lie rank 1. Groups of type ${ }^{2} A_{l},{ }^{2} D_{l},{ }^{3} D_{4},{ }^{2} E_{6}$ are also called by Steinberg groups, groups of type ${ }^{2} B_{2}$ are called by Suzuki groups, groups of type ${ }^{2} G_{2},{ }^{2} F_{4}$ are called by Ree groups, in honor of their discoverers.

The exceptional characteristics of the ground field $F$ for the group $G(F)$ are usually:

- characteristic 2 for types $B_{l}, C_{l}, F_{4},{ }^{2} A_{l},{ }^{2} D_{l}$ and ${ }^{2} E_{6}$;

- characteristics 2 and 3 for types $G_{2}$ and ${ }^{3} D_{4}$.

This is due to the fact that the Coxeter graph associated with the group $G(F)$ has edges of multiplicity 2 or 3 .

In what follows, everywhere the field $F$ is an algebraic extension of the field $K$. The intermediate subgroups between the groups $G(K)$ and $G(F)$ are described in the author's papers [1-3]. For exceptional characteristics, the description depends on whether the field $K$ is perfect. By definition, a field $K$ of characteristic $p>0$ is called perfect if $K^{p}=K$.

*nuzhin2008@rambler.ru

(C) Siberian Federal University. All rights reserved 
In 1983, the following result was obtained in [1]. If the Lie rank of the group $G(F)$ is greater than one and it is different from the group Re of type ${ }^{2} F_{4}$, and in the exceptional characteristics for $G(F)$ the field $K$ is perfect, then the groups lying between the groups $G(K)$ and $G(F)$, are exhausted by the groups $G(P) H$ for some intermediate subfield $P$ and a diagonal subgroup $H$ normalizing the group $G(P)$. We call such intermediate subgroups standard.

In [2], the groups that lie between the Chevalley groups $G(K)$ and $G(F)$ of type $B_{l}, C_{l}$, $F_{4}, G_{2}$ are described in the case of an nonperfect field $K$ that is exceptional characteristics for the group $G(F)$. It turned out that in each of these cases, except type $G_{2}$ in characteristic 2, nonstandard subgroups appear and they are parameterized by two additive subgroups of the field $F$. Moreover, if $G(K)$ is of type $F_{4}$ or $G_{2}$, then both additive subgroups are fields, and if $G(K)$ is of type $B_{l}(l \geqslant 3)$ or $C_{l}(l \geqslant 3)$, then one additive subgroup is a field. The paper [4] contains examples of non-standard intermediate subgroups for types $B_{l}(l \geqslant 3)$ and $C_{l}(l \geqslant 3)$, which are parameterized by two additive subgroups, one of which is not a field, and for the type $B_{2}=C_{2}$ both such additive subgroups may not be fields.

In [3], the groups lying between twisted Chevalley groups $G(K)$ and $G(F)$ of type ${ }^{2} A_{l},{ }^{2} D_{l}$, ${ }^{2} E_{6},{ }^{3} D_{4}$ are described in the case of nonperfect fields $K$ of exceptional characteristic for the group $G(F)$. It turned out that except, perhaps, the type ${ }^{2} D_{l}$, the intermediate subgroups are standard.

In this note, we classify pairs of additive subgroups that parameterize non-standard subgroups between the groups $G(K)$ and $G(F)$ (Section 2) and prove the standardness of such intermediate subgroups for the type ${ }^{2} D_{l}$ (Section 3). Thus, non-standard groups lying between the groups $G(K)$ and $G(F)$ appear only for Chevalley groups of normal type $B_{l}, C_{l}, F_{4}$ and $G_{2}$ over the nonperfect field $F$ of characteristic 2 and, respectively 3 . Note also that if we remove the condition of algebraicity of the extension of a larger field over a smaller one, then the description of intermediate subgroups becomes immeasurable for Chevalley groups associated with Coxeter graphs without multiple connections $[5,6]$.

\section{Pairs of additive subgroups associated with intermediate subgroups of Chevalley groups of type $B_{l}, C_{l}, F_{4}$ и $G_{2}$}

Let $\Phi$ be a reduced indecomposable root system, $\Phi(F)$ be a Chevalley group of type $\Phi$ over the field $F$ generated by the root subgroups

$$
x_{r}(F)=\left\{x_{r}(t) \mid t \in F\right\}, \quad r \in \Phi .
$$

Following V. M. Levchuk [7], by a carpet of type $\Phi$ over $F$, we mean a family of additive subgroups $\mathfrak{A}=\left\{\mathfrak{A}_{r} \mid r \in \Phi\right\}$ of the field $F$ with the condition

$$
C_{i j, r s} \mathfrak{A}_{r}^{i} \mathfrak{A}_{s}^{j} \subseteq \mathfrak{A}_{i r+j s}, \quad r, s, i r+j s \in \Phi, \quad i, j>0,
$$

where $\mathfrak{A}_{r}^{i}=\left\{a^{i} \mid a \in \mathfrak{A}_{r}\right\}$, and constants $C_{i j, r s}$ are equal to $\pm 1, \pm 2$ or \pm 3 . Inclusions (1) come from the Chevalley commutator formula

$$
\left[x_{s}(u), x_{r}(t)\right]=\prod_{i, j>0} x_{i r+j s}\left(C_{i j, r s}(-t)^{i} u^{j}\right), \quad r, s, i r+j s \in \Phi .
$$

Every carpet $\mathfrak{A}$ defines a carpet subgroup $\Phi(\mathfrak{A})$ generated by the subgroups $x_{r}\left(\mathfrak{A}_{r}\right), \quad r \in \Phi$. A carpet $\mathfrak{A}$ is called closed if its carpet subgroup $\Phi(\mathfrak{A})$ has no new root elements, i.e., if

$$
\Phi(\mathfrak{A}) \cap x_{r}(F)=x_{r}\left(\mathfrak{A}_{r}\right) .
$$


Summing Theorems 3.1 and 4.1 from [2], we obtain the following result.

Theorem 1 ([2]). Let $F$ be an algebraic extension of an nonperfect field $K$ of characteristic $p$ and $M$ be a group lying between Chevalley groups $\Phi(K)$ and $\Phi(F)$ of type $\Phi=B_{l}(l \geqslant 2)$, $C_{l}(l \geqslant 2), F_{4}, G_{2}$. Let $p=2$ for $\Phi=B_{l}, C_{l}, F_{4}$ and $p=3$ for $\Phi=G_{2}$. Then $M$ is the product of the carpet subgroup $\Phi(\mathfrak{A})$ and some diagonal subgroup $H_{M}$ normalizing $\Phi(\mathfrak{A})$. The carpet $\mathfrak{A}=\left\{\mathfrak{A}_{r} \mid r \in \Phi\right\}$ is closed and

$$
\mathfrak{A}_{r}= \begin{cases}P, & \text { if } r \text { is a short root }, \\ Q, & \text { if } r \text { is a long root }\end{cases}
$$

for some additive subgroups $P$ and $Q$ of the field $F$ with the conditions

$$
R \leqslant P^{p} \leqslant Q \leqslant P \leqslant K
$$

Moreover, depending on the type of the Chevalley group $\Phi(K)$, the following refinements hold for the additive subgroups $P$ and $Q$ of the field $F$ and the diagonal subgroup $H_{M}$ :

a) if $\Phi=B_{l}$ and $l \geqslant 3$, then $Q$ is a field;

b) if $\Phi=C_{l}$ and $l \geqslant 3$, then $P$ is a field;

c) if $\Phi=F_{4}, G_{2}$, then both additive subgroups $P$ and $Q$ are fields and $H_{M}$ is the unit subgroup.

Here, for any additive subgroup $A$ of some field, by definition

$$
\begin{gathered}
A^{p}=\left\{t^{p} \mid t \in A\right\}, \\
A^{-1}=\{0\} \cup\left\{t \in A \mid t^{-1} \in A\right\} .
\end{gathered}
$$

For $\Phi=F_{4}, G_{2}$, the structure of the additive subgroups $P$ and $Q$ is clear, they are fields. The next proposition clarifies their structure for $\Phi=B_{l}, C_{l}$. For any root $r \in \Phi$ and any $t$ from the multiplicative group $F^{*}$ of the fields $F$ by definition

$$
\begin{gathered}
n_{r}(t)=x_{r}(t) x_{-r}\left(-t^{-1}\right) x_{r}(t), \\
h_{r}(t)=n_{r}(t) n_{r}(-1) .
\end{gathered}
$$

Proposition 1. Let $M, P$ and $Q$ be the same as in Theorem 1 and $p=2$. Then the additive subgroups $P$ and $Q$ satisfy the following conditions:

A1) $1 \in P \cap Q$;

A2) $P Q \leqslant P$;

A3) $P^{2} Q \leqslant Q$;

A4) $P^{2} P \leqslant P$;

A5) $Q^{2} Q \leqslant Q$;

A6) $P^{-1}=P$;

A7) $Q^{-1}=Q$.

Moreover, $P^{2}$ and $Q^{2}$ are fields, $P$ and $Q$ are $P^{2}$-modules, and the subgroup $M$ contains all diagonal elements of the form $h_{r}(t u), t, u \in P \backslash\{0\}$ (respectively, $t, u \in Q \backslash\{0\}$ ), if $r$ is a short root (respectively, if $r$ is a long root). 
Proof. Condition A1) follows from definition of the subgroup $M$. Conditions A2) and A3) follow from the commutator formula (2) and the carpet condition for the subgroup $M$. In [1, p. 535] it was established that for any $t \in P /\{0\}$ (respectively $t \in Q /\{0\}$ ) the polynomial ring $K[t]$ (respectively $K^{2}[t]$ ) lies in $P$ (respectively, in $Q$ ). Hence, since the extension $F / K$ is algebraic, we obtain equalities A6) and A7). For any short root $r$ and any $t, u \in P /\{0\}$, equality A6) implies that $h_{r}(t) h_{r}(u)=h_{r}(t u) \in M$. Similarly, for any long root $r$ and any $t, u \in Q /\{0\}$ from A7), we obtain the inclusion $h_{r}(t u) \in M$. Conjugating the subgroup $x_{r}\left(\mathfrak{A}_{r}\right), r \in \Phi$, by these diagonal elements, we obtain the inclusions A4) and A5). It follows from A4) and A5) that $P^{2}$ and $Q^{2}$ are fields. Finally, from A3) and A4) we obtain that $P$ and $Q$ are $P^{2}$-modules. The proposition is proved.

In [4, Sec. 7] for types $B_{l}(l \geqslant 2)$ and $C_{l}(l \geqslant 2)$, examples of subgroups $P$ and $Q$ from Theorem 1, one of which is not a field, and for the type $B_{2}=C_{2}$ both of which are not fields, are given. Therefore, the inclusion of diagonal elements of the form $h_{r}(t u), t, u \in P \backslash\{0\}$ (respectively, $t, u \in Q \backslash\{0\}$ ) if $r$ is a short root (respectively, if $r$ is a long root) into the subgroup $M$, despite the fact that the product $t u$ may not lie in the subgroup $P$ (respectively, in $Q$ ).

Any algebraic extension of a perfect field is perfectly [8, p. 217] and any finite field is perfect, so the results of the paper [1] say that there are no finite additive subgroups that are not fields that parameterize intermediate subgroups in groups of Lie type. The next proposition asserts that they do not exist even under weaker constraints.

Proposition 2. If the characteristic of the field $F$ is equal to 2 and its finite additive subgroup $P$ satisfies the conditions $\mathrm{A} 1)$ and $\mathrm{A} 4)$, then $P$ is finite field.

Proof. The inclusions A1) and A4) imply the inclusion $P^{2} \leqslant P$, and since squaring is an isomorphism of any field of characteristic 2, taking into account the finiteness of $P$, we obtain the equality $P^{2}=P$. Hence and again in view of A4), $P$ is a ring and, therefore, a field, since any finite integral domain is a field. The proposition is proved.

\section{Groups lying between twisted Chevalley groups}

Let $A$ be a subset of the field $F$. The sets $A^{n}$ and $A^{-1}$ have the same meaning as in Section 2 . The Steinberg group $G(F)$ of type ${ }^{n} X_{l}$ is associated with an automorphism $\sigma$ of order $n$ of the fields $F$. By $F_{\sigma}$ we denote the subfield of fixed elements of the automorphism $\sigma$. By definition, we set $\sigma(u)=\bar{u}, \bar{A}=\{\bar{u} \mid u \in A\}$ and $A_{\sigma}=A \cap F_{\sigma}$. The groups lying between the Steinberg groups $G(K)$ and $G(F)$, where $F$ is an algebraic extension of a nonperfect field $K$ of exceptional characteristic $p$, are described by the author in [3].

Theorem $2([3])$. Let $M$ be a group lying between the Steinberg groups $G(K)$ and $G(F)$ of type ${ }^{2} A_{l}, l \geqslant 4,{ }^{2} D_{l}, l \geqslant 3,{ }^{2} E_{6}$ or ${ }^{3} D_{4}$, where $F$ is an algebraic extension of an nonperfect field $K$ of characteristic $p$, and $p=2$ or 3 if $G(F)$ is of type ${ }^{3} D_{4}$, and $p=2$ otherwise. Then:

1) If $G(F)$ is of type ${ }^{2} A_{l}, l \geqslant 4,{ }^{2} E_{6}$ or ${ }^{3} D_{4}$, then $M=G(P) H_{M}$ for some intermediate subfield $P, K \subseteq P \subseteq F$, and some diagonal subgroup $H_{M}$ normalizing the group $G(P)$.

2) If $G(F)$ is of type ${ }^{2} D_{l}, l \geqslant 3$, then $M=G(P, Q) H_{M}$ for some diagonal subgroup $H_{M}$ normalizing the group $G(P, Q)$ which is generated by intersections

$$
M \cap x_{R}(K)=x_{r}\left(\mathfrak{A}_{r}\right), r \in{ }^{2} D_{l},
$$

where

$$
\mathfrak{A}_{r}= \begin{cases}P, & \text { if } r \text { short root }, \\ Q, & \text { if } r \text { long root }\end{cases}
$$


$P$ and $Q$ are subgroups of the additive group of the field $F$ containing the subfield $K$ and respectively $K_{\sigma}$, and they satisfy the following conditions: $P Q \subseteq P, P^{2} P \subseteq P, P^{-1}=\bar{P}=P$, $u \bar{u}, u+\bar{u} \in Q$ for all $u \in P$, and if $l \geqslant 4$, then $Q$ is a field.

The next proposition axiomatizes the properties of the additive subgroups $P$ and $Q$ from Theorem 2 for $G(F)$ of type ${ }^{2} D_{l}, l \geqslant 3$.

Proposition 3. Let $M, P$ and $Q$ be the same as in Theorem 2 for $G(F)$ of type ${ }^{2} D_{l}, l \geqslant 3$. Then the additive subgroups $P$ and $Q$ satisfy the following conditions:

B1) $1 \in P \cap Q, P \nless F_{\sigma} u Q \leqslant F_{\sigma}$;

B2) $P Q \leqslant P$;

B3) $u \bar{u} t, \bar{u} v+u \bar{v} \in Q$ for any $u, v \in P$ and $t \in Q$;

B4) $P^{2} P \leqslant P$;

B5) $Q^{2} Q \leqslant Q$;

B6) $P^{-1}=P$;

B7) $Q^{-1}=Q$.

Next, we need the following technical lemma on algebraic extensions fields.

Lemma 1. Let $F$ be an algebraic extension of the field $K$, the field $F$ has an automorphism $\sigma$, and $F_{\sigma}$ and $K_{\sigma}$ be centralizers of the automorphism $\sigma$ in the fields $F$ and $K$, respectively. Then the extension $F_{\sigma} / K_{\sigma}$ is also algebraic.

Proof. Let $f$ be an arbitrary nonzero element from $F_{\sigma}$. Since the extension $F / K$ is algebraic, there exists a smallest natural number $m$ such that

$$
f^{m}+k_{m-1} f^{m-1}+\cdots+k_{1} f+k_{0}=0
$$

for some simultaneously non-zero elements $k_{i}$ from the field $K$. But then

$$
f^{m}+\sigma\left(k_{m-1}\right) f^{m-1}+\cdots+\sigma\left(k_{1}\right) f+\sigma\left(k_{0}\right)=0 .
$$

Subtracting the second equality from the first, we obtain

$$
\left(k_{m-1}-\sigma\left(k_{m-1}\right)\right) f^{m-1}+\cdots+\left(k_{1}-\sigma\left(k_{1}\right)\right) f+\left(k_{0}-\sigma\left(k_{0}\right)\right)=0 .
$$

Hence, either for some $i \geqslant 1$ the difference $\left(k_{i}-\sigma\left(k_{i}\right)\right)$ is nonzero, which is impossible due to the minimality of $m$, or all the differences $\left(k_{i}-\sigma\left(k_{i}\right)\right)$ are zero, and then the element $f$ is algebraic over the field $K_{\sigma}$, as required. The lemma is proved.

Proposition 4. Suppose that a field $F$ of characteristic 2 has an automorphism $\sigma$ of order 2, $P$ and $Q$ are its additive subgroups satisfying conditions $\mathrm{B} 1)-\mathrm{B} 7)$. Then the subgroups $P$ and $Q$ are fields, and $Q=P_{\sigma}$.

Proof. Since $Q \leqslant F_{\sigma}$, the inclusion $Q \leqslant P_{\sigma}$ follows from B1) and B2). Since $P \& F_{\sigma}$, then there is an element $t \in P$ such that the sum $t+\bar{t}$ is nonzero and due to B3) lies in $Q$, and in force B7) $\frac{1}{t+\bar{t}} \in Q$. Hence and by virtue of B2) the element $u=\frac{t}{t+\bar{t}}$ lies in $P$, and $u+\bar{u}=1$. Let $v \in P_{\sigma}$. Then by virtue of B3) the subgroup $Q$ contains the element $u \bar{v}+\bar{u} v=u v+\bar{u} v=(u+\bar{u}) v=v$. Therefore, $P_{\sigma} \leqslant Q$. So $Q=P_{\sigma}$. Now B2) implies the inclusion $Q Q \leqslant Q$. Therefore, $Q$ is a 
ring. By virtue of Lemma 1 , the extension $F_{\sigma} / K_{\sigma}$ is algebraic, and since the ring $Q$ is enclosed between $K_{\sigma}$ and $F_{\sigma}$, it is a field.

Let us show that $P$ is a field. Since the extension $F / K$ is algebraic, it suffices to show that for any two elements of $P$ their product lies in $P$. So, let $u, v \in P$. If one of the elements $u$ or $v$ lies in $P_{\sigma}$, then by condition B2) and the equality $Q=P_{\sigma}$ proved above, we obtain the inclusion $u v \in P$. Let both $u$ and $v$ not lie in $Q$. Then they are the roots of the irreducible polynomials $x^{2}+(u+\bar{u}) x+u \bar{u}$ and, accordingly, $x^{2}+(v+\bar{v}) x+v \bar{v}$ of degree 2 over the field $Q$. The polynomial ring $Q[u]$ is a field and, by B2) and B4), lies in $P$. If $v \in Q[u]$, then $u v \in P$. If $v \notin Q[u]$, then the polynomial ring $Q[u, v]$ is an algebraic extension of degree 4 of the field $Q$ and again, by B2) and B4), lies in $P$. Therefore, in any case, $u v \in P$. The proposition is proved.

Combining Theorem 2 and Proposition 4, we obtain the following theorem, which gives a uniform and standard description of intermediate subgroups for Steinberg groups over nonperfect fields in exceptional characteristics.

Theorem 3. Let $M$ be a group lying between the Steinberg groups $G(K)$ and $G(F)$ of type ${ }^{2} A_{l}, l \geqslant 4,{ }^{2} D_{l}, l \geqslant 3,{ }^{2} E_{6}$ or ${ }^{3} D_{4}$, where $F$ is an algebraic extension of an nonperfect field $K$ of characteristic $p$, and $p=2$ or 3 if $G(F)$ is of type ${ }^{3} D_{4}$, and $p=2$ otherwise. Then $M=G(P) H_{M}$ for some intermediate subfield $P, K \subseteq P \subseteq F$, and some diagonal subgroup $H_{M}$ normalizing the group $G(P)$.

This work is supported by the Krasnoyarsk Mathematical Center and financed by the Ministry of Science and Higher Education of the Russian Federation in the framework of the establishment and development of regional Centers for Mathematics Research and Education (Agreement no. 075-02-2020-1534/1) and RFBR (project 19-01-00566).

\section{References}

[1] Ya.N.Nuzhin, Groups contained between groups of Lie type over different fields, Algebra $i$ Logika, 22(1983), no. 5, 526-541 (in Russian).

[2] Ya.N.Nuzhin, Intermediate groups of Chevalley groups of type $B_{l}, C_{l}, F_{4}, G_{2}$ over nonperfect fields of characteristic 2 and 3, Siberian Math. J., 54(2013), 119-123.

DOI: $10.1134 /$ S0037446613010151

[3] Ya.N.Nuzhin, Groups lying between Steinberg groups over non-perfect fields of characteristics 2 and 3, Trudy Inst. Mat. Mekh. UrO RAN, 19(2013), no. 3, 245-250 (in Russian).

[4] Ya.N.Nuzhin, A.V.Stepanov, Subgroups of Chevalley groups of types $B_{l}$ and $C_{l}$ containing the group over a subring, and corresponding carpets, St. Petersburg Math. J., 28(2020), no. 4. Translated from: Algebra i Analiz, 31(2019), no. 4, 198-224 (in Russian).

[5] A.V.Stepanov, Nonstandard subgroups between $\mathrm{E}_{n}(R)$ and $\mathrm{GL}_{n}(A)$, Algebra Colloq., 11(2004), no. 3, 321-334.

[6] A.V.Stepanov, Free product subgroups between Chevalley groups $\mathrm{G}(\Phi, F)$ and $\mathrm{G}(\Phi, F[t])$, J. Algebra, 324(2010), no. 7, 1549-1557.

[7] V.M.Levchuk, Parabolic subgroups of certain ABA-groups, Math. Notes, 31(1982), no. 4, 509-525 (in Russian). 
[8] S.Lang, Algebra, Mir, Moscow, 1968 (translation into Russian).

\section{О парах аддитивных подгрупп, ассоциированных с промежуточными подгруппами групп лиева типа над несовершенными полями}

Яков Н. Нужин

Сибирский федеральный университет Красноярск, Российская Федерация

Аннотация. Ранее (Труды ИММ УрО РАН, 19(2013), № 3) автор описал группы, лежащие между скрученными группами Шевалле $G(K)$ и $G(F)$ типа ${ }^{2} A_{l},{ }^{2} D_{l},{ }^{2} E_{6},{ }^{3} D_{4}$ в случае, когда большее поле $F$ является алгебраическим расширением меньшего несовершенного поля $K$ исключительной характеристики для группы $G(F)$ (характеристики 2 и 3 для типа ${ }^{3} D_{4}$ и только 2 для остальных типов). Оказалось, что кроме, быть может, типа ${ }^{2} D_{l}$, такие промежуточные подгруппы стандартны, то есть они исчерпываются группами $G(P) H$ для некоторого промежуточного подполя $P, K \subseteq P \subseteq$ $F$ и диагональной подгруппы $H$, нормализующей группу $G(P)$. В данной заметка установлено, что промежуточные подгруппы являются стандартными и для типа ${ }^{2} D_{l}$.

Ключевые слова: группы лиева типа, несовершенное поле, промежуточные подгруппы, ковер аддитивных подгрупп. 